\title{
Le cycle évolutif de \\ Morerastrongylus andersoni (Petter, 1972)
}

\author{
par Annie J. PETTER \\ Laboratoire de Zoologie (Vers) associé au C.N.R.S. ( $\mathrm{P}^{r}$ A.-G. ChaBaud), \\ Muséum national d'Histoire naturelle, 43, rue Cuvier, F 75231 Paris Cedex 05
}

\section{Résumé}

Etude du cycle évolutif de Morerastrongylus andersoni (Petter, 1972), Angiostrongylinae parasite de Tatera et Taterillus, Rongeurs Gerbillidés africains. Les larves du premier stade peuvent se développer jusqu'au stade infestant chez Limnaea stagnalis, Planorbarius corneus, Helix aspersa, Deroceras reticulatum, Arion hortensis.

Chez Limnaea stagnalis, à la température de $22^{\circ} \mathrm{C}$, la première mue a lieu après 8 jours et la deuxième mue après 11 jours.

Chez l'hôte définitif, les larves du troisième stade gagnent le foie, vraisemblablement par la cavité générale, en 14 heures, puis les poumons, qu'elles ont à peu près toutes atteints en 24 heures; la troisième mue a lieu entre le $2^{\mathrm{e}}$ et le $3^{\mathrm{e}}$ jour d'infestation, et la quatrième mue entre le $5^{\circ}$ et le $6^{\circ}$ jour; les parasites se localisent dans les bronchioles terminales et les alvéoles, et les œufs effectuent tout leur développement dans les alvéoles. Les Rongeurs parasités commencent à émettre des larves après 24 jours d'infestation.

\section{Summary}

Life-cycle of Morerastrongylus andersoni (Petter, 1972).

Life History of Morerastrongylus andersoni (Petter, 1972) an Angiostrongylinae parasite of the african Gerbillidae Tatera and Taterillus. The development from first larval stage to infective stage can be obtained in Limnaea stagnalis, Planorbarius corneus, Helix aspersa, Deroceras reticulatum, Arion hortensis.

In Limnaea stagnalis, at a temperature of $22^{\circ} \mathrm{C}$, the first molt occurs after eight days and the second one after eleven days.

In the definitive host, the third stage larvae reach the liver, probably through the body cavity, in fourteen hours time, and then the lungs, where the majority of the larvae can be found at twenty-four hours. The third molt occurs between day two and day three after infection and the fourth one between day five and day six. The parasites are localized in 
alveoli and bronchioles, and eggs undergo their development in alveoli. The first larvae are passed in the faeces of parazitised rodents twenty-four days after infection.

La découverte du rôle des larves d'Angiostrongylus cantonensis (Chen, 1935) dans les méningites à éosinophiles en Asie et dans les îles du Pacifique (Rosen et coll., 1961 et 1962 ; Horio et Alicata, 1961 ; Alicata, 1961, 1962) a attiré l'attention sur l'importance des cycles évolutifs des Nématodes Angiostrongylinae. Cette importance a été soulignée plus récemment par la découverte d'une espèce, Morerastrongylus costaricensis (Morera et Cèspedes, 1971), responsable à l'état adulte de lésions dans la cavité abdominale de l'homme.

Nous étudions ici le cycle évolutif de Morerastrongylus andersoni, Angiostrongylinae parasite de Rongeurs gerbillidés africains, décrit par nous en 1972 et placé ultérieurement par Chabaud (1973) dans un nouveau genre, Morerastrongylus.

L'étude du cycle a été réalisée à partir de larves récoltées dans les crottes d'un Tatera cf. nigrita Wroughton, 1906, capturé à Moundou (Tchad) par le $\mathrm{D}^{\mathrm{r}} \mathrm{F}$. Petter en février 1972.

Le $\mathrm{D}^{\mathrm{r}}$ J.-C. Guillon, de l'Institut Pasteur, nous a aidé dans l'interprétation des coupes histologiques et nous l'en remercions vivement.

\section{Développement des oufs.}

Des coupes histologiques de poumons de Rongeurs parasités montrent que les parasites adultes sont localisés dans les bronchioles et les alvéoles (fig. $1 \mathrm{G}$ ). Dans les utérus des femelles mûres, les œufs sont au stade unicellulaire, leur diamètre est d'environ $15 \mu$; la coque est très mince et beaucoup plus grande que l'œuf, formant autour de lui une enveloppe membraneuse très déformable (fig. $2 \mathrm{D}$ ).

La segmentation et l'embryogenèse s'effectuent dans les alvéoles des poumons de l'hôte (fig. $1 \mathrm{H}$ ), où nous trouvons tous les stades du développement jusqu'à l'éclosion des larves du premier stade; l'œuf augmente beaucoup de volume au cours de la segmentation et occupe toute la coque, qui prend une forme ovalaire régulière ; ses dimensions sont de $40 \mu$ sur $30 \mu$ (fig. $2 \mathrm{E}$ ).

\section{Développement dans l'hôte intermédiaire.}

Nous avons utilisé comme hôte intermédiaire des Limnaea stagnalis L., provenant d'un élevage du Laboratoire de Malacologie du Muséum. Les Mollusques sont infestés en les plaçant pendant quelques heures dans un cristallisoir contenant des crottes de Tatera infesté.

A) Mode de pénétration des larves.

Le mode de pénétration des larves de Metastrongyloidea dans les Mollusques est un sujet qui a été très discuté. En ne considérant que la sous-famille des Angiostrongylinae, d'après Hamilton (1969), la pénétration se fait par le pied chez Aelurostrongylus abstrusus (Railliet) ; d'après Drozdz, Doby et Mandahl-Barth (1971), elle se 
fait par ingestion chez Parastrongylus dujardini (Drozdz et Doby) ; les deux modes d'infestation sont possibles d'après Anderson (1962) chez Aelurostrongylus pridhami (Anderson) et, d'après Cheng et Alicata (1965) et Courdurier, Guillon et Malardé (1967), chez Parastrongylus cantonensis (Chen).

Les quelques expériences que nous avons faites chez Morerastrongylus andersoni sont en faveur d'une pénétration par ingestion: nous avons fixé pour l'histologie deux lots de quatre jeunes Limnées, l'un après quatre heures, l'autre après huit heures de contact avec des crottes de Tatera infesté, et les quelques larves que nous avons retrouvées se trouvaient toutes dans la lumière du tube digestif.

B) Allure du développement.

L'étude détaillée du développement a été effectuée chez de très jeunes Limnées (4 $\mathrm{mm}$ à $5 \mathrm{~mm}$ de long), dont la transparence rendait facile l'observation des larves par simple écrasement du Mollusque entre lame et lamelle. Une Limnée a été sacrifiée chaque jour à partir du lendemain de l'infestation jusqu'à l'obtention de larves infestantes. L'expérience a été réalisée au mois de mai 1972 à la température du laboratoire (de $22^{\circ} \mathrm{C}$ à $25^{\circ} \mathrm{C}$ ). La plupart des Limnées se sont trouvées infestées, chacune possédant d'une à cinq larves.

- Le lendemain de l'infestation, les larves présentent le même aspect et la même taille que les larves trouvées dans les crottes; pendant les jours qui suivent, elles s'enroulent en spirale, puis elles grandissent et s'épaississent beaucoup, tandis que la paroi de l'intestin se charge de granulations de réserve.

- Nous observons la première mue huit jours après l'infestation; à partir de ce stade, les larves ne grandissent plus.

- Dès le neuvième jour, la cuticule de la deuxième mue se décolle à l'extrémité antérieure; le onzième jour, les larves sont entourées de deux enveloppes de mue décollées tout le long du corps.

C) SPÉCIFICITÉ DE L'HôTE INTERMÉDIAIRE.

Les Limnaea stagnalis ne se rencontrant pas au Tchad, ne sont évidemment pas l'hôte intermédiaire naturel du parasite; nous avons obtenu le développement des larves jusqu'au stade infestant chez d'autres Mollusques aquatiques: Planorbarius corneus (L.), et terrestres : Helix aspersa Müller, Deroceras reticulatum (Müller), Arion hortensis Férussac ; l'hôte intermédiaire est donc peu spécifique, comme c'est généralement le cas chez les Métastrongylides.

\section{Développement dans l'hôte définitif.}

L'étude du développement a été effectuée chez de jeunes Tatera cf. nigrita Wroughton 1906 et Taterillus cf. congicus Thomas 1915, provenant de Moundou (Tchad).

Les Rongeurs ont été infestés en leur introduisant dans la gorge de 150 à 300 larves, récoltées après digestion artificielle des Limnées infestées. 
A) Durée de la période prépatente.

Trois jeunes Tatera sont infestés à la même date et des analyses de selles pratiquées tous les jours à partir du lendemain de l'infestation.

L'un d'eux meurt 23 jours après l'infestation : ses poumons montrent des tuméfactions blanches avec des Nématodes adultes, des œufs embryonnés et quelques larves.

Les deux autres commencent à émettre des larves dans leurs selles le lendemain de ce jour, c'est-à-dire 24 jours après l'infestation. A partir de cette date, tous les examens de crottes montrent des larves très abondantes.

Un autre Tatera infesté ultérieurement commence également à émettre des larves 24 jours après l'infestation.

B) Migrations et ali.ure du développement.

De jeunes Tatera, Taterillus et Souris blanches sont autopsiés après 4 heures, 7 heures, 12 heures, 14 heures, 19 heures, 24 heures, 2 jours, 3 jours, 4 jours, 5 jours, 6 jours, 8 jours et 15 jours après l'infestation :

- 4 heures (Taterillus): Une vingtaine de larves sont trouvées dans l'estomac, 7 larves dans l'intestin grêle, 3 larves dans l'eau où baigne le foie, 1 larve dans l'eau où baignent les reins.

- 7 heures (Souris blanche): 9 larves sont trouvées dans l'estomac (contenu et paroi), 1 larve dans l'intestin grêle, 2 larves dans l'eau où baigne le foie, 1 larve dans l'eau où baignent les poumons.

- 12 heures (Taterillus) : Chez cet animal, la cavité abdominale est ouverte et le corps mis à tremper dans un verre à pied pendant une heure avant de détacher les organes: 36 larves sont trouvées dans l'eau de trempage; les organes sont ensuite détachés : 50 larves sont trouvées dans le foie, 16 larves dans les poumons, 16 larves dans l'eau où a baigné le tube digestif non ouvert, 5 larves dans l'eau où a baigné la carcasse, aucune larve dans les contenus intestinal et stomacal.

- 14 heures (Taterillus): De nombreuses larves du troisième stade semblables aux larves infestantes sont trouvées dans le foie, aucune dans les autres organes; l'histologie a été effectuée sur un lobe du foie: nous avons trouvé 2 larves dans des capillaires situés au voisinage d'une veine centrolobulaire (fig. 1 B), 2 larves dans des capillaires situés à la périphérie du lobe et 2 larves situées entre la capsule de Glisson et le parenchyme hépatique (fig. $1 \mathrm{~A}$ ).

- 19 heures (Taterillus) : Chez cet animal, la cavité thoracique est ouverte et le sang prélevé dans le cœur et la veine cave: aucune larve n'est trouvée; le corps est ensuite mis à tremper dans un verre à pied avant de détacher les organes et d'ouvrir la cavité abdominale : aucune larve n'est trouvée dans les eaux de trempage; les organes sont ensuite détachés : 29 larves sont trouvées dans le foie, 1 larve dans l'eau où a baigné la carcasse et 31 larves dans un lobe du poumon; l'autre lobe est fixé pour l'histologie: des larves sont trouvées dans les alvéoles situés à la périphérie du lobe.

- 24 heures (Taterillus): Quelques larves sont trouvées dans le foie et de nombreuses larves dans les poumons, semblables aux larves infestantes; aucune n'est trouvée dans les autres organes. 

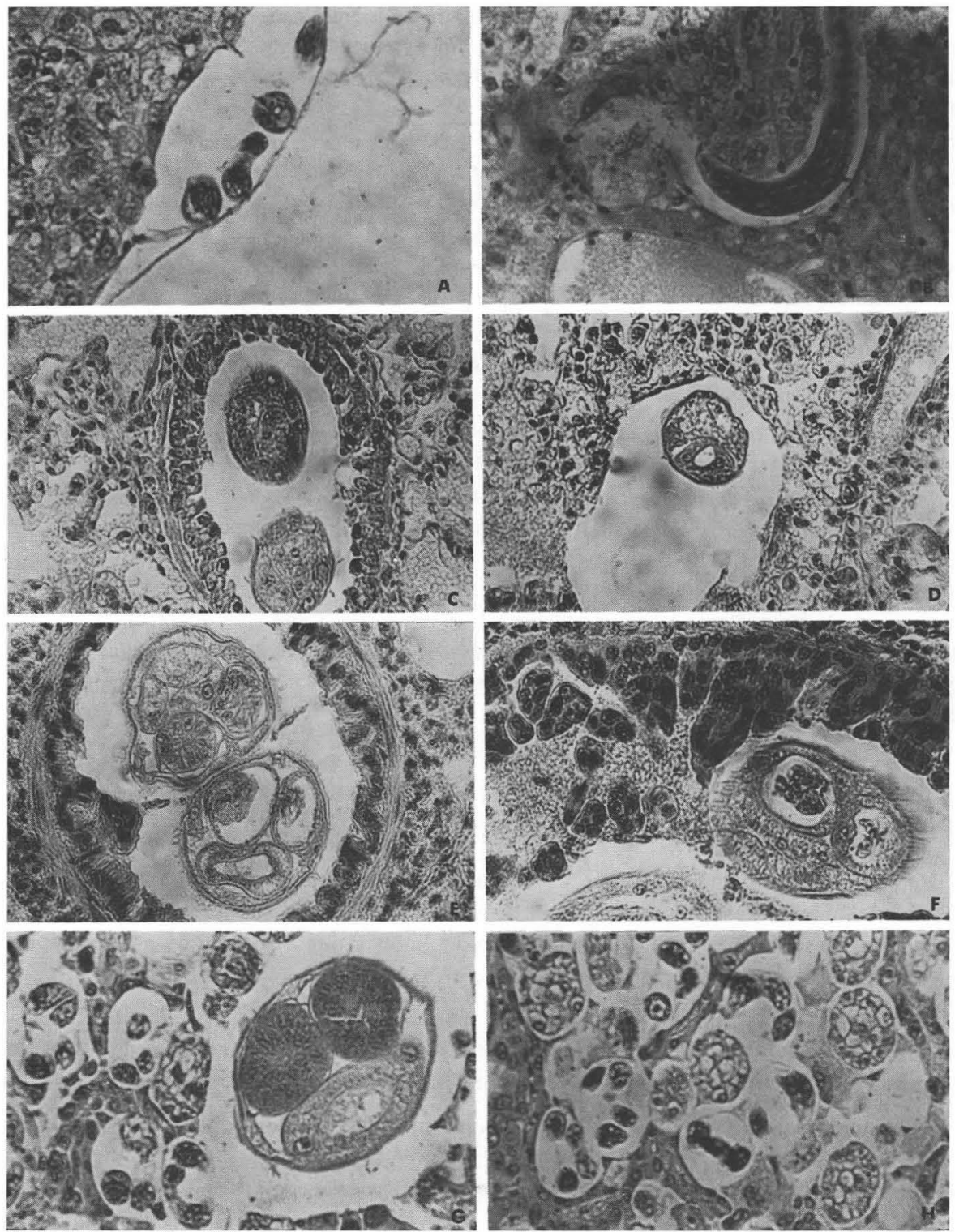

FIG. 1. - A : larve située entre la capsule de Glisson et le parenchyme hépatique (14 heures d'infestation). B : larve située dans un capillaire au voisinage d'une veine centro-lobulaire (14 heures d'infestation). C: adulte juvénile situé dans une bronchiole ( 8 jours d'infestation). D: adulte juvénile situé dans un alvéole pulmonaire (8 jours d'infestation). E, F : adultes situés dans des bronchioles (15 jours d'infestation) montrant la réaction hyperplasique de l'épithélium. G: adulte situé dans un alvéole pulmonaire ( 5 mois d'infestation). $\mathrm{H}$ : œufs en segmentation et larves du $1^{\text {er }}$ stade dans les alvéoles. (Grossissement: 250). 
- 2 jours (Tatera): De nombreuses larves du troisième stade, de même longueur que les larves infestantes, sont trouvées dans les poumons, aucune dans les autres organes; chez toutes les larves examinées (15), la cuticule de la troisième mue est décollée à l'extrémité postérieure.

- 3 jours (Taterillus): Une vingtaine de larves du quatrième stade, longues de $625 \mu$ à $880 \mu$, sont trouvées dans les poumons, aucune dans les autres organes; une larve est entourée de la mue du troisième stade, largement décollée à l'extrémité antérieure.

- 4 jours (Taterillus) : Des larves du quatrième stade, longues de $825 \mu$ à $1.200 \mu$, sont trouvées dans les poumons; chez deux d'entre elles, la quatrième mue commence à se décoller à l'extrémité postérieure.

- 5 jours (Taterillus): Des larves du quatrième stade, longues de $1.100 \mu$ à $1.200 \mu$, sont trouvées dans les poumons; chez la plupart d'entre elles, la cuticule de la quatrième mue est largement décollée aux extrémités.

— 6 jours (Tatera) : Des adultes juvéniles (mâles longs de $1.700 \mu$ à $1.750 \mu$, femelles longues de $2.500 \mu$ à $2.750 \mu$ ) sont trouvés dans les poumons.

- 8 jours (Taterillus) : Des adultes juvéniles (mâles longs de $1.600 \mu$ à $2.800 \mu$, femelles longues de $2.600 \mu$ à $3.700 \mu$ ) sont trouvés dans les poumons ; des coupes histologiques d'un lobe du poumon montrent qu'ils sont localisés dans les bronchioles terminales et les alvéoles (fig. 1 C-D).

— 15 jours (Taterillus) : Des adultes mâles (longs de $5.500 \mu$ à $6.100 \mu$ ) et femelles (longues de $11.700 \mu$ à $15.000 \mu$ ) sont trouvés dans les poumons, localisés dans les bronchioles et les alvéoles; les poumons contiennent des œufs en segmentation disséminés dans le parenchyme pulmonaire (fig. $1 \mathrm{E}-\mathrm{F}$ ).

C) SPÉCIFICITÉ de L’Hôte dÉFINITIF.

Nous avons tenté l'infestation de quelques autres espèces de Rongeurs:

- Un Meriones crassus, né en laboratoire: un premier examen de selles, fait 29 jours après l'infestation, montre de très nombreuses larves ; il continue à émettre des larves jusqu'à sa mort, six mois après l'infestation; ses poumons contiennent 3 femelles et 4 mâles adultes, ainsi que de très nombreux œufs et larves.

- Deux Rats blancs de laboratoire: des examens de selles effectués tous les 8 jours après le $24^{e}$ jour d'infestation sont tous négatifs ; l'un est autopsié après 2 mois d'infestation: aucune larve ni adulte ne sont retrouvés dans les poumons ni dans le foie.

- Quatre Souris blanches de laboratoire infestées en deux lots:

- Des examens de selles effectués après 24 jours d'infestation chez le premier lot ne montrent que de très rares larves. Une Souris est autopsiée après 33 jours : 3 femelles et 3 mâles adultes sont trouvés dans les poumons; les utérus des femelles contiennent des œufs normaux, mais nous trouvons seulement 2 larves écloses dans l'eau où baignent les poumons. Une Souris est autopsiée après 75 jours: les poumons présentent des tuméfactions dans lesquelles se trouvent des mâles et des femelles adultes, de nombreux œufs en développement, mais seulement quelques larves écloses. 
- Des examens de selles effectués après 24 jours chez le deuxième lot montrent des larves abondantes. Une Souris est autopsiée après 40 jours: ses poumons contiennent de nombreux adultes, des œufs en développement et de nombreuses larves. La deuxième Souris meurt après 50 jours : 3 mâles et 2 femelles adultes sont trouvés dans les poumons.

L'infestation réussit donc indifféremment chez trois espèces de Gerbillidae; chez les Souris blanches, les larves infestantes atteignent le stade adulte dans les poumons, mais ces hôtes sont moins favorables, puisque, chez 2 Souris sur 4, très peu d'œufs se développent jusqu'à l'éclosion des larves du premier stade ; les Rats blancs ne semblent pas réceptifs, ce qui avait déjà été constaté par Drozdz et Doby (1970) chez Parastrongylus dujardini.

\section{Morphologie des stades larvaires.}

Premier stade (fig. 2 A-B).

Les larves recueillies dans les selles des Rongeurs ont l'aspect habituel aux larves de Métastrongylides: stoma avec rhabdions chitinisés, œsophage rhabditoïde, queue avec une encoche dorsale, ailes latérales; elles mesurent de $280 \mu$ à $300 \mu$.

Dimensions d'une larve longue de $300 \mu$ : largeur maximum, $15 \mu$; œsophage, $130 \mu$; anneau nerveux et pore excréteur situés à $50 \mu$ et $85 \mu$ de l'extrémité antérieure; ébauche génitale située à $100 \mu$ de l'extrémité postérieure ; queue longue de $20 \mu$, avec un appendice terminal de $8 \mu$; ailes latérales débutant à $18 \mu$ de l'extrémité antérieure et se terminant à $10 \mu$ de la pointe caudale.

Ces larves grandissent et épaississent beaucoup dans les Mollusques ; après 7 jours d'infestation, elles atteignent $550 \mu$ et leur intestin a le même aspect que chez les larves du deuxième stade décrites ci-dessous.

Deuxième STADE (fig. 2 C).

Le deuxième stade est très fugitif, puisque la deuxième mue commence à se décoller dès le lendemain de l'apparition de la première mue.

Les larves mesurent de $450 \mu$. à $550 \mu$, elles sont dépourvues d'ailes latérales et de rhabdions chitinisés à l'extrémité antérieure ; les cellules intestinales sont chargées de granulations de réserve, leurs limites apparaissent nettement.

Dimensions d'une larve longue de $545 \mu$ : largeur maximum, $40 \mu$; œsophage, $160 \mu$; anneau nerveux et pore excréteur situés respectivement à $80 \mu$ et $100 \mu$ de l'extrémité antérieure; ébauche génitale située à $190 \mu$ de l'extrémité postérieure ; queue, $42 \mu$.

TROISIÈME STADE (fig. 3, 4).

Les larves infestantes du troisième stade (fig. 3 A-B-C) ont la même taille que les larves du deuxième stade, mais elles sont plus minces, beaucoup plus mobiles et munies d'ailes latérales; l'extrémité antérieure est munie de rhabdions chitinisés, les limites des 
cellules intestinales ne sont plus visibles, le nombre et la taille des granulations de réserve intestinales ont beaucoup diminué ; en vue médiane, nous avons observé de minuscules déirides.

Dimensions d'une larve longue de $560 \mu$ : largeur maximum, $30 \mu$; œsophage, $170 \mu$; anneau nerveux, déirides et pore excréteur situés respectivement à $90 \mu, 90 \mu$ et $100 \mu$ de l'extrémité antérieure ; ébauche génitale située à $170 \mu$ de l'extrémité postérieure ; queue, $40 \mu$; ailes latérales débutant à $28 \mu$ de l'extrémité antérieure et se terminant à $12 \mu$ de l'extrémité postérieure.
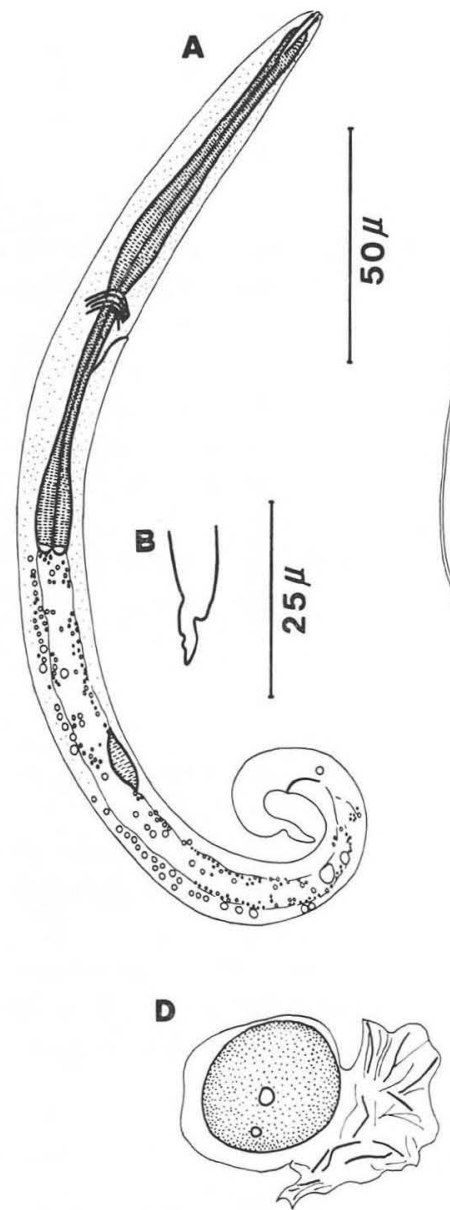
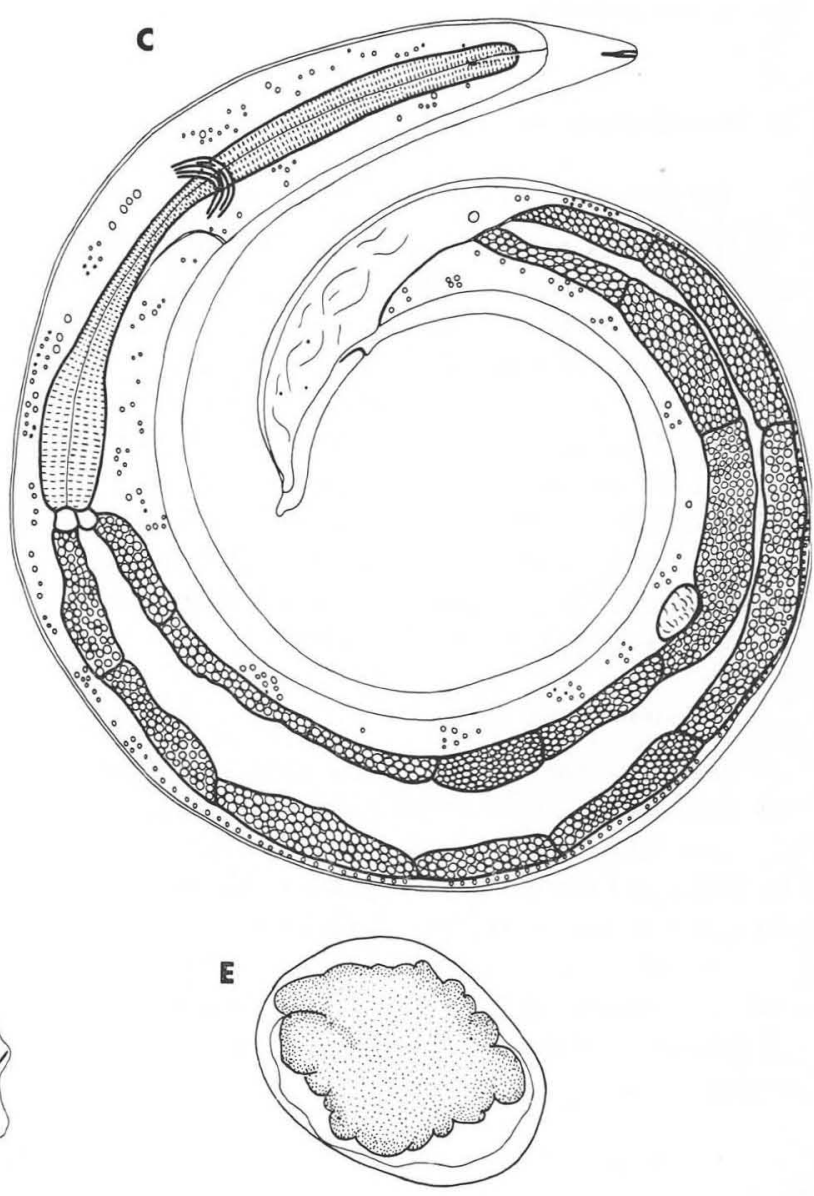

Fig. 2. - A : larve du $1^{\mathrm{er}}$ stade, vue latérale. B : extrémité caudale de la larve du $1^{\mathrm{er}}$ stade. C: larve du $2^{r}$ stade, vue latérale. D: œuf prélevé dans l'utérus d'une femelle. E: œuf en segmentation dans les poumons de 1'hôte. (A, $\mathrm{C}=$ éch. : $50_{\mu} ; \mathrm{B}, \mathrm{D}, \mathrm{E}=$ éch. : $25 \mathrm{u}$ ). 
Les larves du troisième stade trouvées dans les poumons de l'hôte définitif après 2 jours d'infestation ont la même taille que les larves infestantes; les deux sexes peuvent être différenciés d'après la position de l'ébauche génitale (fig. 4 B-C); la vulve étant située très postérieurement chez les femelles adultes, nous supposons que l'ébauche la plus postérieure correspond aux larves femelles; la cuticule est décollée du corps à l'extrémité postérieure et la cavité buccale du quatrième stade est en formation à l'extrémité antérieure (fig. $4 \mathrm{~A}$ ).

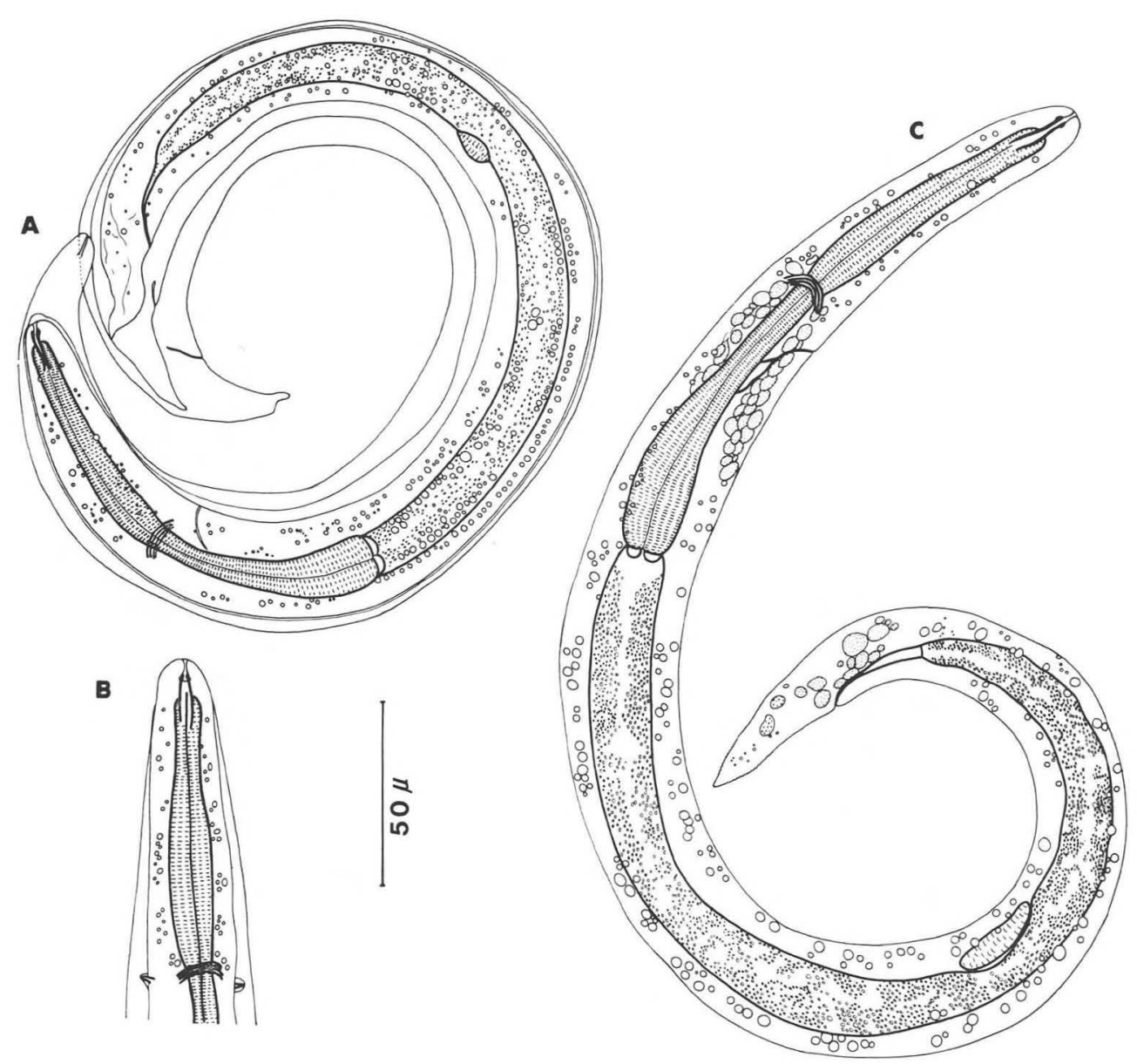

Fig. 3. - Larves infestantes du $3^{\mathbf{e}}$ stade.

A: larve entourée des mues des stades précédents, vue latérale. B: extrémité antérieure de la larve, vue médiane. C: larve débarrassée de ses mues, vue latérale. 
QuATRIÈme STADE (fig. 5).

Les larves du quatrième stade mesurent de $600 \mu$ (les plus jeunes) à $1.200 \mu$ (les plus âgées) ; elles sont dépourvues d'ailes latérales ; la paroi du stoma est épaissie par deux anneaux chitinoïdes superposés (fig. 5 B) ; l'œsophage est de forme légèrement rhabditoïde ; les glandes excrétrices subventrales sont bien visibles; la queue se termine par deux petits lobes arrondis (fig. $5 \mathrm{C}$ ).
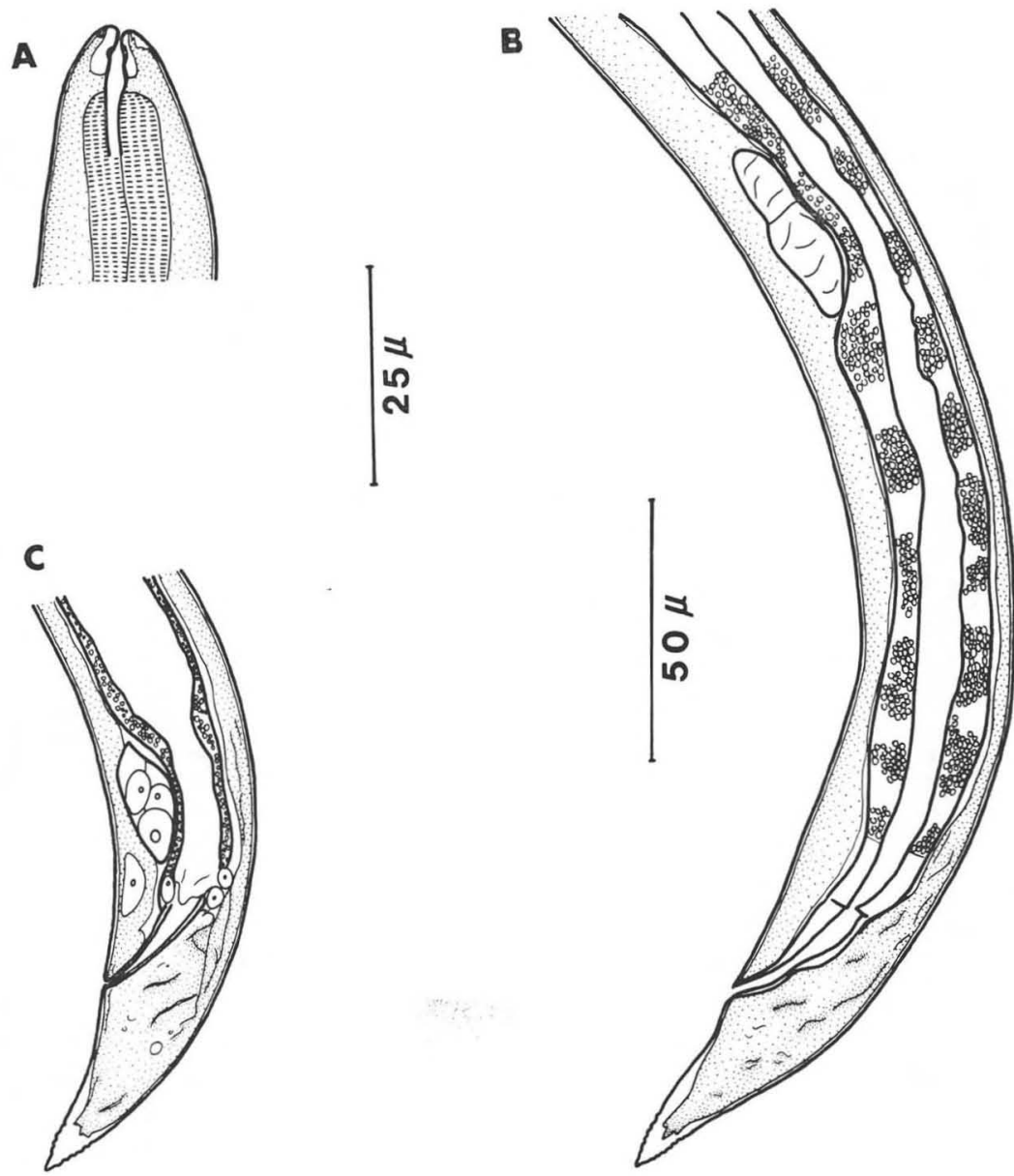

Fig. 4. - Larves du $3^{\text {e }}$ stade recueillies après 2 jours d'infestation chez l'hôte définitif.

A: extrémité antérieure montrant la cavité buccale du $4^{e}$ stade en formation. B: région postérieure d'une larve mâle (?). C: région postérieure d'une larve femelle (?). ( $\mathrm{A}=$ éch. $25 \mu ; \mathrm{B}, \mathrm{C}=$ éch : $50 \mu)$. 
Chez les larves jeunes (3 jours d'infestation) (fig. 5 A), l'ébauche génitale est petite et en forme de fuseau, la queue est identique dans les deux sexes.

Chez les larves âgées (4 jours et 5 jours d'infestation) (fig. 5 E-F), l'ébauche génitale s'est allongée en cordon, le vagin cuticulaire est différencié chez les larves femelles (fig. 5 F) ; la queue est de forme différente suivant le sexe (fig. 5 G-H).

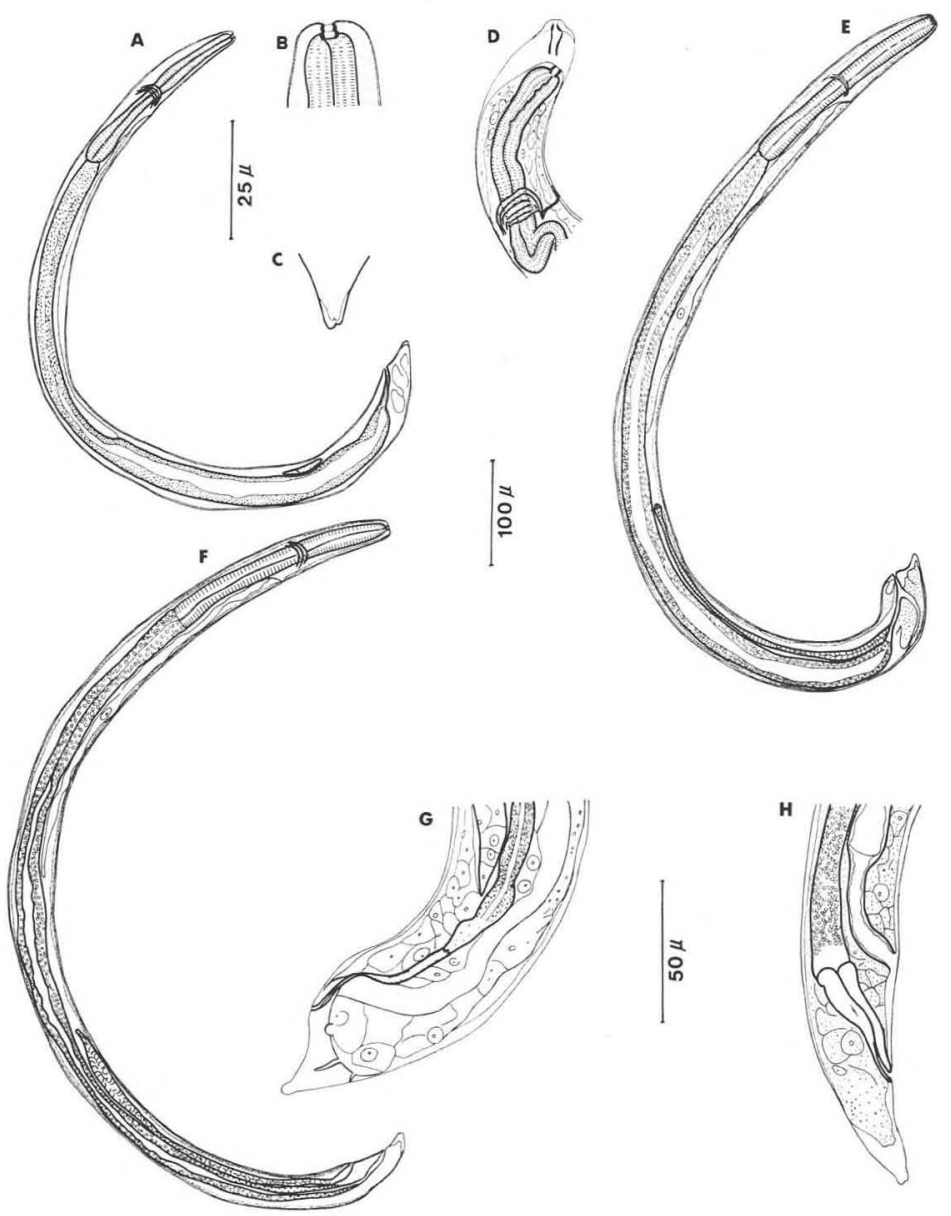

FIG. 5. - Larves du $4^{\mathrm{e}}$ stade.

A, B, C, D : larves recueillies après 3 jours d'infestation chez l'hôte définitif. A: larve entière, vue latérale. B: extrémité antérieure. C: extrémité postérieure. D: extrémité antérieure d'une larve en train d'effectuer la $3^{\mathrm{e}}$ mue. E, F : larves recueillies après 4 jours d'infestation. $\mathrm{E}$ : larve mâle, vue latérale. $\mathrm{F}$ : larve femelle, vue latérale. $\mathrm{G}, \mathrm{H}$ : larves recueillies après 5 jours d'infestation. G: région postérieure d'une larve mâle. $\mathrm{H}$ : région postérieure d'une larve femelle. (B, $\mathrm{C}=$ éch. : $25 \mu ; \mathrm{A}, \mathrm{E}, \mathrm{F}$ : éch. : $100 \mu ; \mathrm{D}, \mathrm{G}, \mathrm{H}=$ éch. : $50 \mu$ ) 
Dimensions d'une jeune larve longue de $880 \mu$ : largeur maximum, $40 \mu$; œsophage, $190 \mu$; pore excréteur et anneau nerveux situés respectivement à $100 \mu$ et $120 \mu$ de l'extrémité antérieure ; ébauche génitale située à $160 \mu$ de l'extrémité postérieure ; queue, $35 \mu$.

Dimensions d'une larve femelle âgée, longue de $1.160 \mu$ : largeur maximum, $50 \mu$; œsophage, $230 \mu$; anneau nerveux et pore excréteur situés respectivement à $90 \mu$ et $110 \mu$ de l'extrémité antérieure; queue, $20 \mu$; ébauche vulvaire située à $55 \mu$ de l'extrémité postérieure.

Dimensions d'une larve mâle âgée, longue de $1.200 \mu$ : largeur maximum, $50 \mu$; œsophage, $225 \mu$; anneau nerveux et pore excréteur situés respectivement à $100 \mu$. et $120 \mu$ de l'extrémité antérieure; queue, $30 \mu$.

\section{Conclusions.}

\section{Allure du Cycle Évolutif.}

Nous pouvons donc retracer ainsi les grandes lignes du cycle évolutif :

Chez les Limnaea stagnalis, et à la température de $22^{\circ} \mathrm{C}$, les larves du premier stade grandissent rapidement (de $300 \mu$ à $550 \mu$ ), tout en se chargeant de granulations de réserve; la première mue a lieu après 8 jours et la deuxième mue après 11 jours ; les deuxième et troisième stades ne grandissent pas chez les Mollusques.

Ingérées par un hôte favorable, les larves du troisième stade gagnent le foie, où nous les retrouvons à peu près toutes après 14 heures d'infestation; il semble qu'elles se rendent directement à cet organe par la cavité générale, sans emprunter la voie sanguine, puisque, d'une part, nous retrouvons un grand nombre de larves dans cette cavité après 12 heures d'infestation et que, d'autre part, certaines larves ont été trouvées localisées entre la capsule de Glisson et le parenchyme hépatique.

Elles gagnent ensuite les poumons qu'elles ont à peu près toutes atteints après 24 heures; là encore, la localisation des larves dans les alvéoles de la périphérie du poumon après 19 heures d'infestation semble indiquer qu'elles s'y rendent directement sans emprunter la voie sanguine ; sans avoir subi d'accroissement de taille, elles effectuent la troisième mue entre le $2^{\mathrm{e}}$ et le $3^{\mathrm{e}}$ jour d'infestation ; en 4 jours, les quatrièmes stades doublent leur taille (de $600 \mu$ à $1.200 \mu$ ), tandis que les organes génitaux s'organisent.

La quatrième mue a lieu entre le $5^{\mathrm{e}}$ et le $6^{\mathrm{e}}$ jour d'infestation; les cinquièmes stades grandissent rapidement dans les bronchioles terminales et les alvéoles; quinze jours après l'infestation, ils ont atteint la taille adulte; les œufs s'embryonnent dans les alvéoles des poumons et les Rongeurs commencent à émettre des larves dans leurs selles après 24 jours d'infestation.

Le développement dans l'hôte définitif est maintenant connu chez un certain nombre de Metastrongyloidea et les processus de ce développement se montrent très 
divers, tant par l'organe où s'effectuent les mues que par les voies de migration jusqu'à la localisation de l'adulte.

Chez les Angiostrongylinae, le développement a été élucidé chez six espèces, et on observe trois modalités de développement différentes:

Le cas le plus fréquent est celui où les mues s'effectuent dans les poumons après une migration des larves du troisième stade. Chez notre espèce et chez Parastrongylus dujardini (Drozdz et Doby, 1970) (voir Drozdz et Doby, 1970), cette migration comporte un séjour dans le foie ; ce type de développement est également présenté chez les Skrjabingylinae par Crenosoma vulpis (Dujardin, 1845) (voir Stockdale et Hulland, 1970) ; chez les deux dernières espèces, les auteurs supposent que la migration jusqu'au foie s'effectue par la circulation sanguine, bien que les larves n'aient jamais été trouvées dans la veine porte; nous avons vu que, chez notre espèce, il semble que cette hypothèse soit fausse et que les larves arrivent directement au foie. La migration s'effectue sans passage dans le foie chez Aelurostrongylus pridhami Anderson, 1962 (voir Stockdale, 1970) et Aelurostrongylus abstrusus (Railliet, 1898) (voir Hobmaier et Hobmaier, 1935).

Chez Angiostrongylus vasorum (Baillet), les mues s'effectuent dans les ganglions lymphatiques abdominaux, et ce sont les jeunes cinquièmes stades qui migrent jusqu'aux poumons (voir Guilhon et Cens, 1969 ; Rosen et coll., 1970).

Enfin, chez Parastrongylus cantonensis, les mues s'effectuent dans le système nerveux central (voir Mackerras et Sandars, 1955 ; Jindrak, 1968) ; cette espèce présente donc un type de cycle exceptionnel chez les Angiostrongylinae ; cependant, le rôle des autres espèces en Pathologie humaine ne doit pas être écarté a priori, car des migrations aberrantes sont toujours possibles chez des hôtes accidentels.

\section{Bibliographie}

Alicata (J. E.), 1961. - A cause of parasitic meningitis in the Pacific-rat lungworms. Hawaï Farm. Bur. J., 1-2 (Special Number).

—, 1962. - Angiostrongylus cantonensis (Nematoda : Metastrongylidae) as a causative agent of eosinophilic meningitis of man in Hawaï and Tahiti. Can. J. Zool., 40, 5-8.

ANDERSON (R. C.), 1962. - The systematics and transmission of new and previously described Metastrongyles (Nematoda: Metastrongylidae) from Mustela vison. Can. J. Zool., 40, 893-920.

Chabaud (A.-G.), 1972. - Description de Stefanskostrongylus dubosti, n. sp., parasite du Potamogale et essai de classification des Nématodes Angiostrongylinae. Ann. Parasit. hum. comp., 47, 735-744.

Cheng (T. C.) et Alicata (J.E.), 1965. - On the mode of infection of Achatina fulica by the larvae of the Angiostrongylus cantonensis. Malacologia, 2, 267-274.

Courdurier (J.), Guillon (J.-C.) et Malarde (L.), 1967. - Réalisation du cycle d'Angiostrongylus cantonensis (Chen) au laboratoire. II. Utilisation d'Australorbis glabratus (Say) comme hôte intermédiaire. Contribution à l'étude du mode d'infestation du 
Mollusque par les larves du parasite. Aspects histologiques. Bull. Soc. Path. Exo., $60,165-173$.

Drozdz (J.) et Doby (J.-M.), 1970. - Evolution morphologique, migrations et chronologie du cycle d'Angiostrongylus (Parastrongylus) dujardini Drozdz et Doby 1970 (Nematoda: Metastrongyloidea) chez ses hôtes définitifs. Bull. Soc. Scient. Bretagne, 45, 229-239.

- - - et Mandahl-Barth (G.), 1971. - Etude des morphologie et évolution larvaires d'Angiostrongylus (Parastrongylus) dujardini Drozdz et Doby 1970 (Nematoda: Metastrongyloidea). Infestation des Mollusques hôtes intermédiaires. Ann. Parasit. hum. comp., 46, 265-276.

Guilhon (J.) et Cens (B.), 1969. - Migrations et évolution d'Angiostrongylus vasorum (Baillet, 1866) dans l'organisme du chien. C.R. Acad. Sci. (Paris), Sér. D, 269, 2377-2380.

Hamilton (J. M.), 1969. - On the migration, distribution, longevity and pathogenicity of larvae of Aelurostrongylus abstrusus in the snail, Helix aspersa. J. Helm., 43, 319-325.

Hobmaier (M.) et Hobmaier (A.), 1935. - Mammalian phase of the lungworm Aelurostrongylus abstrusus in the cat. J. Am. Vet. Med. As., 87, 191-198.

Horio (S. R.) et Alicata (J. E.), 1961. - Parasitic meningo-encephalitis in Hawaï, a new parasitic disease of man. Hawaï Med. J., 21, 139-140.

JINDRAK (K.), 1968. - Early migration and pathogenicity of Angiostrongylus cantonensis in laboratory rats. Ann. Trop. Med. Parasit., 62, 506-517.

Mackerras (M. J.) et Sandars (D. F.), 1955. - The Life-history of the rat Lungworm, Angiostrongylus cantonensis (Chen) (Nematoda: Metastrongylidae). Austral. J. Zool., 3, 1-21.

Morera (P.) et Cespedes (R.), 1971. - Angiostrongylus costaricensis n. sp. (Nematoda : Metastrongyloidea), a new lungworm occuring in man in Costa Rica. Rev. Biol. Trop., 18, 173-185.

Petter (A.-J.), 1972. - Description d'une nouvelle espèce d'Aelurostrongylus parasite de Rongeur africain. Ann. Parasit. hum. comp., 47, 131-137.

Rosen (L.), Ash (L. R.) et Wallace (G. D.), 1970. - Life-history of the canine lungworm Angiostrongylus vasorum (Baillet). Am. J. Vet. Res., 31, 131-143.

—, Chapell (R.), Laqueur (G. L.), Waillace (G. D.) et Weinstein (P. P.), 1962. - Eosinophilic meningoencephalitis caused by a metastrongylid lungworm of rats. J. Amer. Med. Assoc., 179, 620-624.

-, Laigret (J.) et Bories (S.), 1961. - Observations on an outbreak of eosinophilic meningitis on Tahiti, French Polynesia. Amer. J. Hyg., 74, 26-41.

Stockdale (P. H. G.), 1970. - The development, route of migration, and pathogenesis of Perostrongylus pridhami in mink. J. Parasit., 56, 559-566.

- et Hulland (T. J.), 1970. - The pathogenesis, route of migration and development of Crenosoma vulpis in the dog. Pathologia vet., 7, 28-42. 